ARTICLE PREPRINT

\title{
bioR $\chi$ iv

\section{Atto 465 derivative is a nuclear stain with unique excitation and emission spectra for multiplex immunofluorescence histochemistry}

\author{
Joshua, T. Dodge ${ }^{1}$, Ana C. Costa-da-Silva ${ }^{1}$, Christopher T. Hogden ${ }^{1}$ Eva Mezey ${ }^{1}$, Jacqueline W. Mays ${ }^{*}$
}

The architecture of a biologic response is inextricably linked with the tissue architecture of the target site. Multiplex immunofluorescence (mIF) is an effective technique for the maximal visualization of multiple target proteins in situ. This powerful tool is limited by the spectral overlap separation of the currently available synthetic fluorescent dyes. The fluorescence excitation wavelengths ranging between $405 \mathrm{~nm}$ and 488nm are rarely used in mIF imaging and serve as a logical additional slot for a fluorescent probe. In the present study, we demonstrate that Atto 465-pentafluoroaniline (Atto 465-p), a derivative of the fluorescent dye Atto 465, can serve as a nuclear stain in the violet-blue region of the visible spectrum. This opens the $405 \mathrm{~nm}$ channel, traditionally used for nuclear stain, for detection of another experimental target. This increases the flexibility of the mIF panel and, with appropriate staining and microscopy, enables the quantitative analysis of at least six targets in one tissue section.

\section{Introduction}

Recent advances in multiplex immunofluorescence (mIF) technology allow the simultaneous detection of multiple target proteins on a single tissue sample. This provides an accurate characterization of different cellular types and states in the context of their spatial relationship within the local environment. Diagnosis, treatment, and a better understanding, of complex diseases, such as cancer (1-3) and neurogenerative diseases $(4,5)$ is made possible via mIF. This sensitive technique can be affected by many factors including cross-reactivity of secondary antibodies and the spectral overlap of fluorophores. Several methods were recently developed to circumvent these limitations and achieve accurate mIF staining $(6,7)$. However, some require expensive, specialized kits and equipment $(7,8)$.

The tyramide signal amplification (TSA) system is based on a horseradish peroxidase (HRP) catalyzed deposition of fluorophore-labelled tyramide on and near a target protein or nucleic acid $(9,10)$. This straightforward technique is highly sensitive and specific, enables the precise detection of low-abundance proteins and the use of same-species antibodies $(11,12)$ and is cost-effective. Although a variety of target species and fluorophores are commercially available (12), it is rare for researchers to make use of the functional excitation wavelengths between 405 and $488 \mathrm{~nm}$.

Atto $465 \mathrm{NHS}$ ester, hereafter referred to as Atto 465 , is struc- turally similar to proflavine (13). During the course of work to use Atto 465 to label antibodies for mIF panels, it became apparent that the dye was acting instead as an effective nuclear stain. In this study, we demonstrate that Atto 465 stains nuclei and is cleanly imaged at $465 \mathrm{~nm}$. It's derivative, Atto 465-p, has higher photostability than Atto $465 \mathrm{NHS}$ ester and can be easily separated from both $405 \mathrm{~nm}$ and $488 \mathrm{~nm}$ wavelengths using a confocal microscope with a tunable excitation source. We also demonstrate its utility by using Atto 465-p as a nuclear probe in a 6-plex IF assay.

\section{Materials and Methods}

Animals

BALB/cJ female mice ( $8-12$ weeks old) were purchased from The Jackson Laboratory (Bar Harbor, ME). All animal procedures were approved by the Institutional Animal Care and Use Committee of NIDCR under an approved animal protocol and were performed according to the guidelines of the institute.

\section{Human tissues}

Human minor salivary gland (MSG) and oral mucosa (OM) were obtained from healthy volunteers enrolled on registered, IRB-approved protocol NCT03602599 in accordance with the Declaration of Helsinki. Formalin-fixed paraffin-embedded (FFPE) tonsils, kidney, and skin blocks were ob-

\footnotetext{
1National Institute of Dental and Craniofacial Research, National Institutes of Health, Bethesda, MD *To whom correspondence should be addressed. Jacqueline W. Mays DDS, MHSc, PhD; BIdg 30, Rm 303; MSC 4340; 30 Convent Drive; Bethesda, MD 20892, USA; Jacqueline.Mays@nih.gov
} 


\section{ARTICLE PREPRINT}

tained from a commercial tissue bank. All tissues were fixed in formalin, embedded in paraffin, and serially cut into $5 \mu \mathrm{m}$ thick sections onto charged slides. Human karyotype slides were donated from the National Human Genome Research Institute Cytogenetic and Microscopy Core.

\section{Atto 465 NHS ester - crosslinking reaction}

Atto 465 NHS ester (Sigma Aldrich, Saint Louis, MO, PubChem Substance ID 329757879) was resuspended in DMSO, aliquoted and lyophilized. After resuspension in 1\% triethylamine DMSO, 2,3,4,5,6-Pentafluoroaniline, Sigma-Aldrich) was added to the lyophilized dye in $10 \%$ molar excess. Each reaction was brought to a $16 \mathrm{mM}$ concentration of Atto 465-NHS ester and stirred at room temperature (RT) overnight on a vibrating plate protected from light. Dye was diluted to a final volume of $50 \mathrm{uL}$ with $\mathrm{DMSO}$, aliquoted and stored at $-20 \circ C$.

\section{Steady-state absorption and fluorescence measurements}

Absorption measurements were performed on a Nanophotometer N60 (Implen, Westlake Village, CA). Fluorescence emission spectra were measured on a SpectraMax i3 (Molecular Devices, San Jose, CA). The dye was diluted to 160 $\mathrm{uM}$, and a blank with equal moles of reactants (minus the dye) was used. Samples were excited at $430 \mathrm{~nm}$ and the fluorescent intensity was measured from $455-650 \mathrm{~nm}$. All data were normalized to the highest fluorescent or absorption value.

\section{Photobleaching, photoconverting, and imaging}

FFPE human tonsils were deparaffinized in xylene substitute (Sigma-Aldrich, St. Louis, MO), rehydrated in a graded ethanol series and stained for 10 minutes with Atto 465-carboxcylic acid (8 uM; Sigma-Aldrich, St. Louis, MO), Atto 465-p $(8 \mu \mathrm{M})$, or YoPro-1 $(0.125 \mu \mathrm{M}$; Invitrogen, Grand Island, NY). Tissue sections were imaged using a Nikon A1R confocal microscope Nikon A1R confocal microscope (fitted with a Pan Fluor 40x/1.30 oil objective) using the NIS Elements imaging software (Nikon Instruments Inc., Melville, NY). Photobleaching was achieved through repetitive imaging every 34 seconds for 2.66 hours using a $486 \mathrm{~nm}$ laser set to $60 \%$ power. Laser energy varies between microscopes, so the microscope settings were converted into energy density to ensure results could be reproduced by other microscopes. The energy density was calculated by measuring the laser power in millijoules ( $\mathrm{mJ}$ ) at the back focal plane of the objective, at the given pixel dwell and resolution, with a compact power and energy meter (Thorlabs Newton, $\mathrm{NJ}$ ), then multiplying by the pixel dwell time ( $\mu \mathrm{s} /$ pixel), to generate the total millijoules per pixel of each scan, or area per pixel ( $\mu \mathrm{m} 2 /$ pixel) to get the final energy density per scan. This was performed similar to Doyle et. al (14). The confocal line average was set to 8 in Galvano mode to ensure the laser was constantly delivering power to the tissue throughout the 2.66 hours time-lapse. All images were captured at $1024 \times 1024$ pixels with a 1.1-pixel dwell time ( $\mu \mathrm{s} / \mathrm{pixel})$. To capture the $405 \mathrm{~nm}$ channel, the $405 \mathrm{~nm}$ laser was set to $2 \%$ power. Thus, any effect the $405 \mathrm{~nm}$ laser had in reversing the $486 \mathrm{~nm}$ photobleaching was minimal. Autofocus was used to prevent focus drift over time. For photoconversion experiments, the $486 \mathrm{~nm}$ laser was set to $3 \%$ power and a time-lapse was run for 12 hours with 20-minute imaging intervals.

\section{Zinc and formalin fixed tissue staining}

Submandibular gland, oral mucosa, and lymph node from BALB/cJ mice and humans were harvested and either fixed for $48 \mathrm{~h}$ at RT in 10\% neutral buffered formalin (Sigma-Aldrich, St. Louis, MO) or zinc-fixation buffer ( $\mathrm{pH} 6.5$ - 7), prepared as described (15). Tissues were processed, embedded in paraffin and serially cut at $5 \mu \mathrm{m}$ thick sections. For staining, sections were deparaffinized in xylene substitute (Sigma-Aldrich, St. Louis, MO), and rehydrated in a decreasing ethanol series. Tissues were then stained with Atto 465-p $(4 \mu \mathrm{M})$, Hoechst 33342, (16.2 $\mu \mathrm{M}$; Invitrogen, Eugene, OR), and/or ToPro-3-3 (1 $\mu \mathrm{M}$; Thermo-Fisher Scientific, Waltham, MA) for 10 minutes at RT, protected from light, followed by washes in phosphate buffered saline (PBS; Corning, Manassas, VA).

\section{Frozen section IHC}

Mice lymph nodes were dissected, embedded in Optimum Cutting Temperature compound (OCT; Sakura, Torrance, $\mathrm{CA}$ ) and flash-frozen in cooled 2-methylbutane (Sigma-Aldrich, St. Louis, MO). Five $\mu$ m-thick cryosections were cut onto charged slides and stored at $-80^{\circ} \mathrm{C}$ until use. For staining, sections were air-dried, rehydrated in PBS and fixed at $-200 \mathrm{C}$ for $5 \mathrm{~min}$ in 1:1 mixture of methanol and acetone. After washing with PBS, tissues were stained with Atto 465-p $(4 \mu \mathrm{M})$, Hoechst $33342(16.2 \mu \mathrm{M})$, and/or ToPro-3-3, $(1 \mu \mathrm{M})$ for 10 minutes at RT, protected from light, followed by serial washes with PBS.

\section{Mouse peritoneal exudate cell staining}

BALB/cJ mice were euthanized, and cells were collected by peritoneal lavage with $5 \mathrm{~mL}$ ice-cold DMEM medium (Gibco, Life Technologies, Grand Island, NY). Peritoneal exudate cells were centrifuged at $200 \mathrm{xg}$ and $40 \mathrm{C}$, for 10 minutes. Total cell numbers were determined using an automated cell counter (Countess II FL, Thermo Fisher Scientific). Cells were plated onto eight-well chamber slides (Nunc, Rochester, NY) after resuspension in DMEM supplemented with 50 $\mathrm{IU} / \mathrm{mL}$ penicillin $\mathrm{G}, 50 \mu \mathrm{g} / \mathrm{mL}$ streptomycin and $10 \%$ fetal calf serum at a density of $1 \times 106$ cells $/ \mathrm{mL}$. Cells were kept in an incubator at $37^{\circ} \mathrm{C}$ and $5 \% \mathrm{CO} 2$ for $3 \mathrm{~h}$, washed with Hank's Balanced Salt Solution and fixed in 4\% paraformaldehyde (PFA) for 30 minutes at RT. After washing with PBS, cells were stained with Atto $465-\mathrm{p}(4 \mu \mathrm{M}, 2 \mu \mathrm{M}, 1 \mu \mathrm{M}$, or $0.5 \mu \mathrm{M})$, Hoechst $33342(16.2 \mu \mathrm{M})$, and/or ToPro-3-3, $(1 \mu \mathrm{M})$ for 10 minutes at RT, protected from light, followed by washes with PBS.

\section{mIF staining}

mIF was performed as described previously (10) using tyramide signal amplification (TSA) and repeated removal of 


\section{ARTICLE PREPRINT}

sequentially applied antibodies (leaving the fluorochrome labelled water insoluble tyramide behind) before the next staining. Briefly, FFPE tonsil specimens were deparaffinized with xylene substitute (Sigma-Aldrich) and rehydrated with decreasing concentrations of ethanol $(100 \%, 95 \%$ and $70 \%$ $\mathrm{vol} / \mathrm{vol}$; Sigma-Aldrich). Antigen retrieval was performed by microwaving slides in $10 \mathrm{mM}$ citric acid buffer $(\mathrm{pH} \mathrm{6})$, and endogenous peroxidase (HRP) activity was blocked using Bloxall (Vector Labs, Burlingame, CA). Primary antibodies, appropriate HRP-conjugated polymers (R\&D Systems, Minneapolis, MN) and tyramide-conjugated fluorophores, provided in Supplementary Tabel 1, were serially added to tissue sections, followed by microwave treatment and blocking steps after each cycle. Nuclei were counterstained with Atto 465-p at $4 \mu \mathrm{M}$ for 10 minutes at RT. Slides mounted with Fluoro-Gel (Electron Microscopy Sciences, Hatfield, PA).

\section{Image acquisition and analysis}

For Atto $465 p$ titration and colocalization studies, tissues or cells were imaged using a Nikon A1R confocal microscope (fitted with a Pan Fluor 40x/1.30 oil objective) using the NIS Elements imaging software (Nikon Instruments Inc., Melville, NY).

mIF staining was imaged using a Leica SP8 confocal microscope (fitted with a HC APO CS2 40x/1.30 oil objective) using the Leica Application Suite X (LAS X) software Leica, Wetzlar, Germany). Images acquired from both Nikon and Leica microscopes were processed using Fiji is Just ImageJ
(Fiji). Image colocalization was quantified using Volocity 3.6 software (Perkin Elmer, Waltham, MA). Threshold for each image was determined automatically according to the Costes method (16). Mander's overlap coefficient was then created for each image, comparing Hoechst 33342 staining to both Atto 465 and ToPro-3-3.

\section{Statistical analysis}

Statistical analysis was performed using GraphPad Prism 8.4.1 software (GraphPad). All values are expressed as mean \pm SEM. Data were compared using repeated measures one-way ANOVA. P-value $<0.05$ is considered statistically significant.

\section{Results}

Synthesis and characterization of Atto 465-p

Atto 465 is structurally similar to proflavine (13), however it has a smaller Stokes shift and an emission spectrum further away from the 488 channel (Figure S1). This allows Atto 465 to be more readily separated from the 488 channel than is proflavine (Figure S1 B-D). In the present work, we tested Atto 465-p, a derivative of the fluorescent dye Atto 465, as a nuclear probe. A schematic diagram of the nucleophilic substitution reaction with Atto 465-NHS ester and 2,3,4,5,6-pentafluoroaniline is proposed in Figure 1A (17). Atto 465-p absorption (left side) and fluorescence spectra (right side) in PBS are depicted in Figure 1B. The absorbance spectrum gave maxima at 439 and $465 \mathrm{~nm}$ and the fluorescence emission extended from 465 to beyond $550 \mathrm{~nm}$ wavelengths.<smiles>Nc1c(F)c(F)c(F)c(F)c1F</smiles><smiles></smiles><smiles>CC(C)(C)[Mg]Br</smiles><smiles>Nc1ccc2ccc3ccc[nH]cc-3n(CCCC(=O)Nc3c(F)c(F)c(F)c(F)c3F)c2c1</smiles>

B

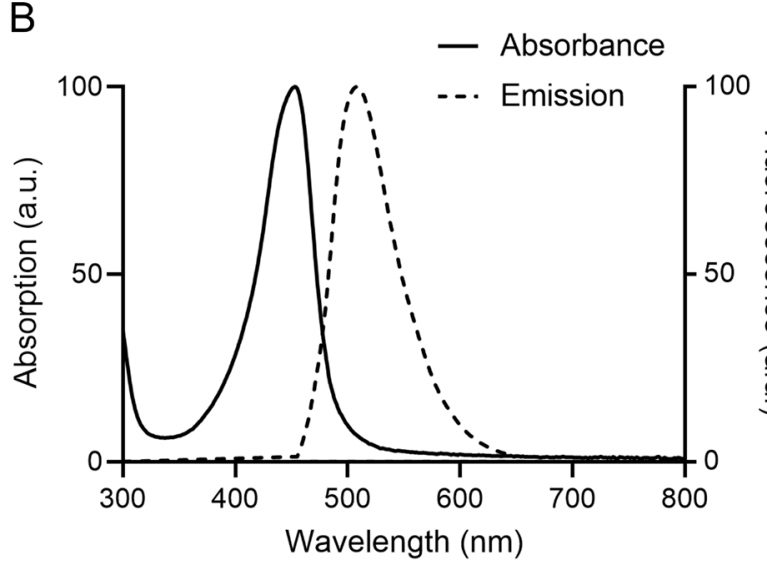

\section{C}

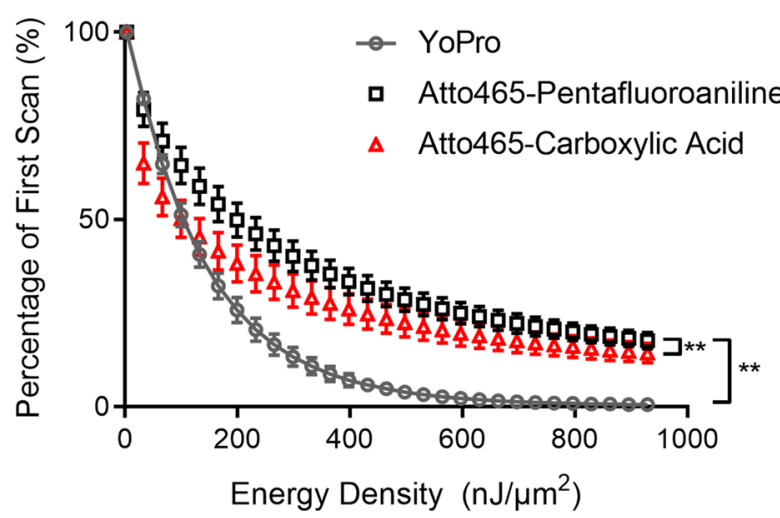

Figure 1: Characterization and photostability of Atto 465-p. A) Scheme illustrating presumed chemical structure and proposed nucleophilic substitution reaction between Atto 465-NHS ester and 2,3,4,5,6 pentafluoroaniline to synthetize Atto 465-p. B) Absorption (solid line) and fluorescence emission (dashed line) spectra of $8 \mu \mathrm{M}$ Atto 465-p in PBS peaking at $450 \mathrm{~nm}$ and $508 \mathrm{~nm}$, respectively. C) Photobleaching kinetics of YoPro-1, Atto 465-p and Atto $465 \mathrm{upon}$ continuous irradiation by $486-n m$ laser. ${ }^{* *}=p<0.0001$ 
bioRxiv preprint doi: https://doi.org/10.1101/2021.05.14.444234; this version posted May 15, 2021. The copyright holder for this preprint (which

was not certified by peer review) is the author/funder. This article is a US Government work. It is not subject to copyright under 17 USC 105 and is also made available for use under a CCO license.

\section{ARTICLE PREPRINT}

A

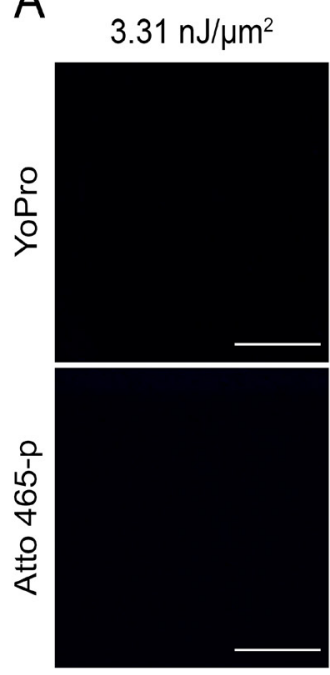

C
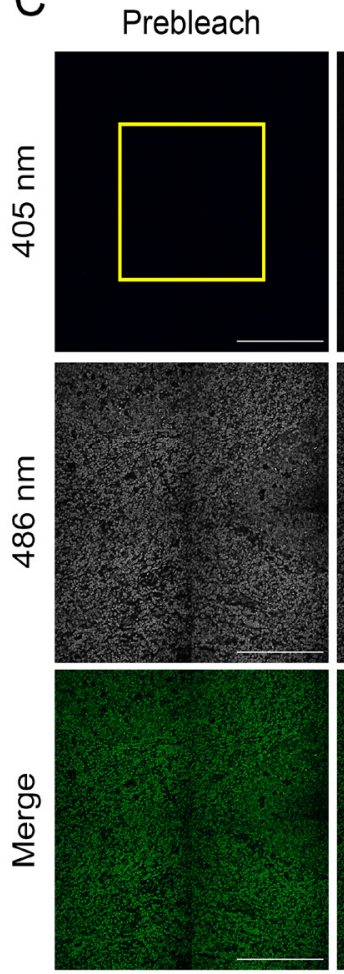

$132.7 \mathrm{~nJ} / \mathrm{um}^{2}$
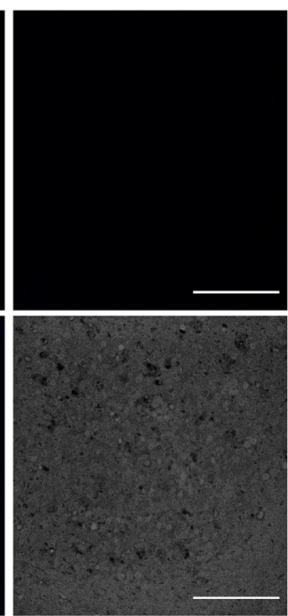

Oh Postbleach
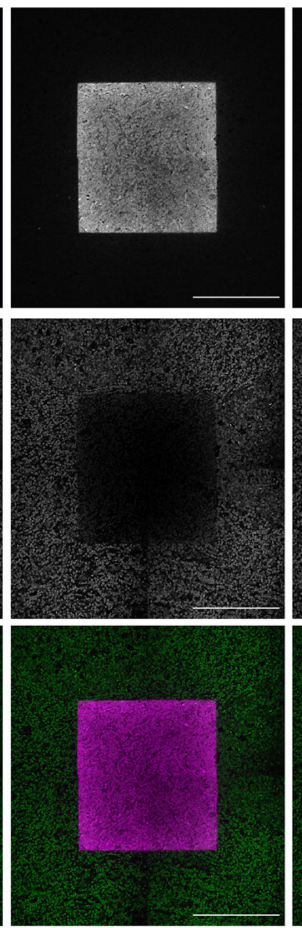

$663.5 \mathrm{~nJ} / \mu \mathrm{m}^{2}$

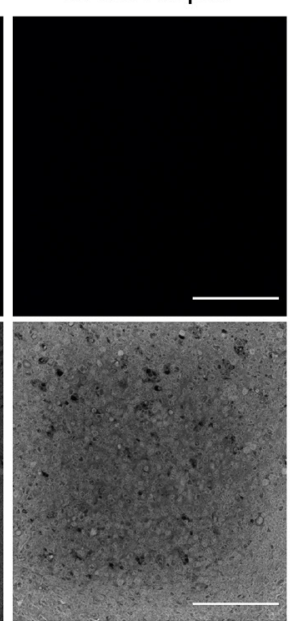

2h Postbleach
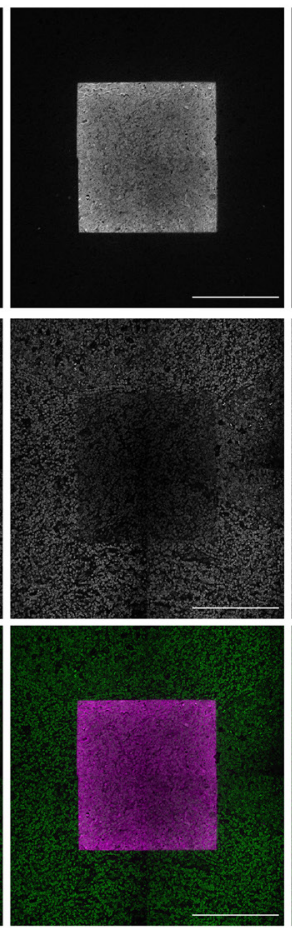

B

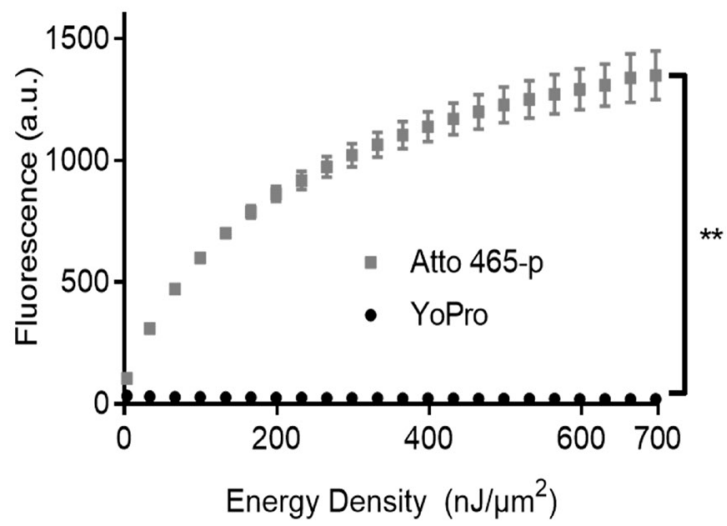

D
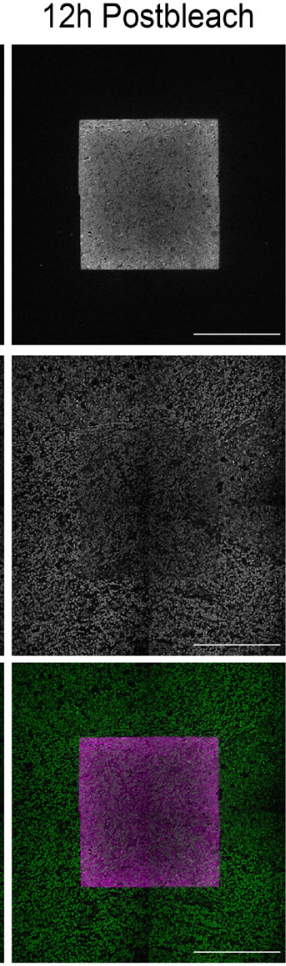

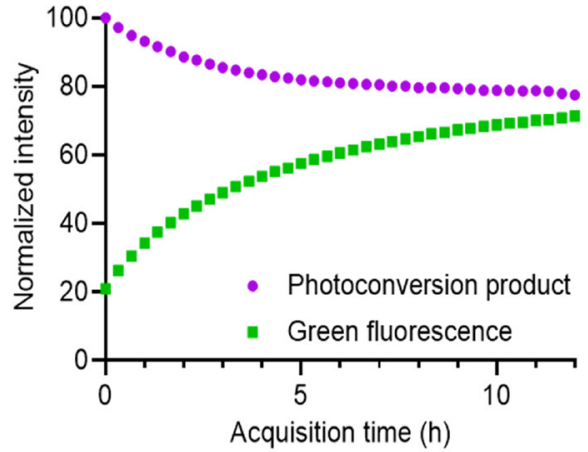

Acquisition time (h)

Figure 2: Characterization of Atto 465-p photoconversion. A) Representative images of human FFPE tonsil stained with YoPro-1 (top; $0.125 \mu \mathrm{M}$ ) or Atto 465-p (bottom; $8 \mu \mathrm{M}$ ) and subjected to photobleaching. Photoconversion product was detectable with illumination at $405 \mathrm{~nm}$. Scale bar=100 $\mu \mathrm{m}$. B) Plot illustrates the correlation between photoconverted fluorescence quantification and laser power density for both Atto 465-p (grey squares) and YoPro-1

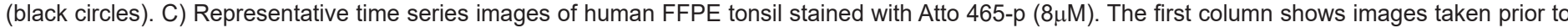
photobleaching (Prebleach); immediately after photobleaching (second column, Oh Postbleach), a 2-hour timepoint (third column, 2h Post-bleach) and 12-hour (fourth column, 12h Postbleach) timepoint using the same image settings as the pre-photobleached image (see Material and Methods). Scale bar=200 $\mu \mathrm{m}$. D) Graph shows the kinetic analysis of the average fluorescence intensities in the bleach ROI (yellow square in C) excited at $405 \mathrm{~nm}$ (magenta dots) and $486 \mathrm{~nm}$ (green squares) immediately after photobleaching (Time $0 \mathrm{~h}$ ). ${ }^{* *}=p<0.0001$, paired t-test. 


\section{ARTICLE PREPRINT}
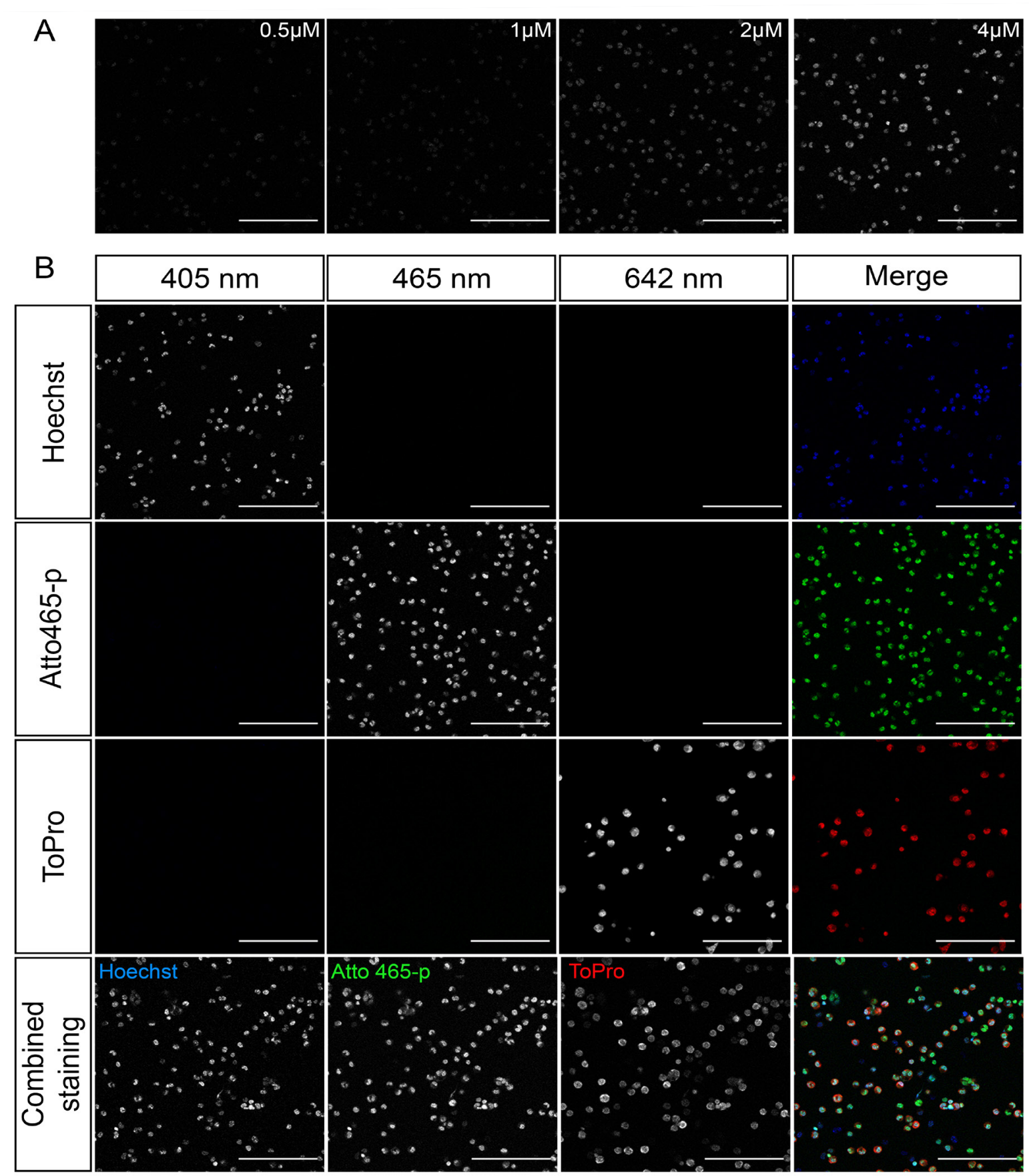

Figure 3: A) Serial dilution staining was done on 4\%PFA fixed mouse peritoneal cells to determine optimal concentration for Atto 465-p. B) Mouse cells stained with either Hoechst 33342, Atto 465-p, ToPro-3, or a combination of all dyes. Scale bar $=50 \mu \mathrm{m}$

revealing the marginal zone $(\mathrm{MZ})$ and germinal center $(\mathrm{GC})$ of a follicle. As expected, CD3, CD8 and cKit showed membranous staining, residing primarily extrafollicularly, whereas CD56 expression was membranous but present both in the extra and intrafollicular space. Moreover, IL-17 cytoplasmic staining was localized primarily outside the germinal center. As a proof of concept, each channel was evaluated individually showing that Atto 465-p can be fluorescently separated from the $405 \mathrm{~nm}$ (IL-17 green) and 488nm (CD8 yellow) channels. Together, these data suggest that Atto 465-p can be added as a nuclear stain to a mIF protocol, thus facilitating the use of the $405 \mathrm{~nm}$ wavelength for another target.

\section{Discussion}

In the present work, we demonstrate that Atto 465-p is a specific and stable fluorescent dye for nuclear staining with excellent performance. It can be used as an alternative to DAPI in mIF assays in mouse and human cells and tissues. These data show comparable nuclear staining between Atto 465-p and other common nuclear dyes Hoechst 33342 and ToPro-3.

Atto 465-p is a green fluorescent dye that absorbs light between 439 and $465 \mathrm{~nm}$ (Figure 1). It is easily excited with an argon laser in instruments emitting 488-nm light, which is a more common imaging component than is the UV laser. The 


\section{ARTICLE PREPRINT}

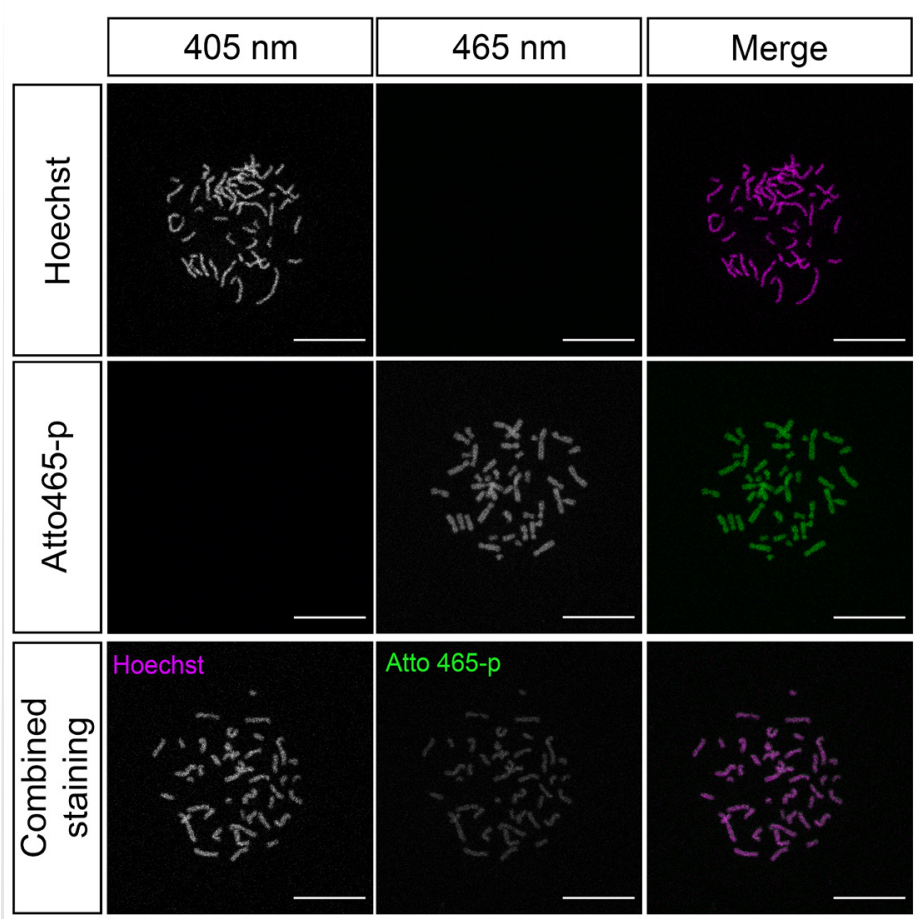

Figure 4: Immunofluorescence staining of human karyotype shows patterning of Hoechst 33342 and Atto 465-p with colocalization in the bottom row, suggesting that Atto 465-p and Hoechst 33342 label chromatin similarly. Scale bar $=25 \mu \mathrm{m}$

Next, we evaluated Atto 465 free dye (carboxylic acid) and Atto 465-p for photobleaching behavior and compared them to a stable green nuclear dye, YoPro-1 (18). Under continuous excitation using $486 \mathrm{~nm}$ laser light, we observed faster reduction in fluorescence signal associated with YoPro-1 and Atto 465 free dye (carboxylic acid) when compared to Atto 465-p (Figure 1C), demonstrating that Atto 465-p has greater photostability. Further characterization revealed that Atto 465-p undergoes a photoconversion from green to a blue-emitting form upon exposure to a $486 \mathrm{~nm}$ light in a dosage dependent manner (Figure 2A and B). However, Atto 465-p regains fluorescence in a few hours, while maintaining the blue fluorescent form (Figure 2B).

\section{Atto 465-p is a nuclei-specific fluorescent dye}

We next evaluated the nuclear localization performance of Atto 465-p in multiple cell and tissue types. We determined an optimal working concentration of Atto 465-p from four different dilutions $(0.5 \mu \mathrm{M}$ to $4 \mu \mathrm{M})$ for fixed peritoneal exudate cells with 10-minute incubation. An efficient fluorescence signal was achieved at $4 \mu \mathrm{M}$ as demonstrated in Figure $3 \mathrm{~A}$. We compared Atto 465-p staining to ToPro-3 and another common nuclear dye, Hoechst 33342. These dyes were selected because they differ in fluorescent spectrum, allowing them to be stained together on the same tissue. The resultant staining shows a similar pattern for Atto 465-p, Hoechst 33342 and ToPro-3 (Figure 3B). Single-color slides were stained to rule out fluorescent bleed through between channels (Figure 3B).

To further confirm Atto 465-p affinity and specificity for nuclei,

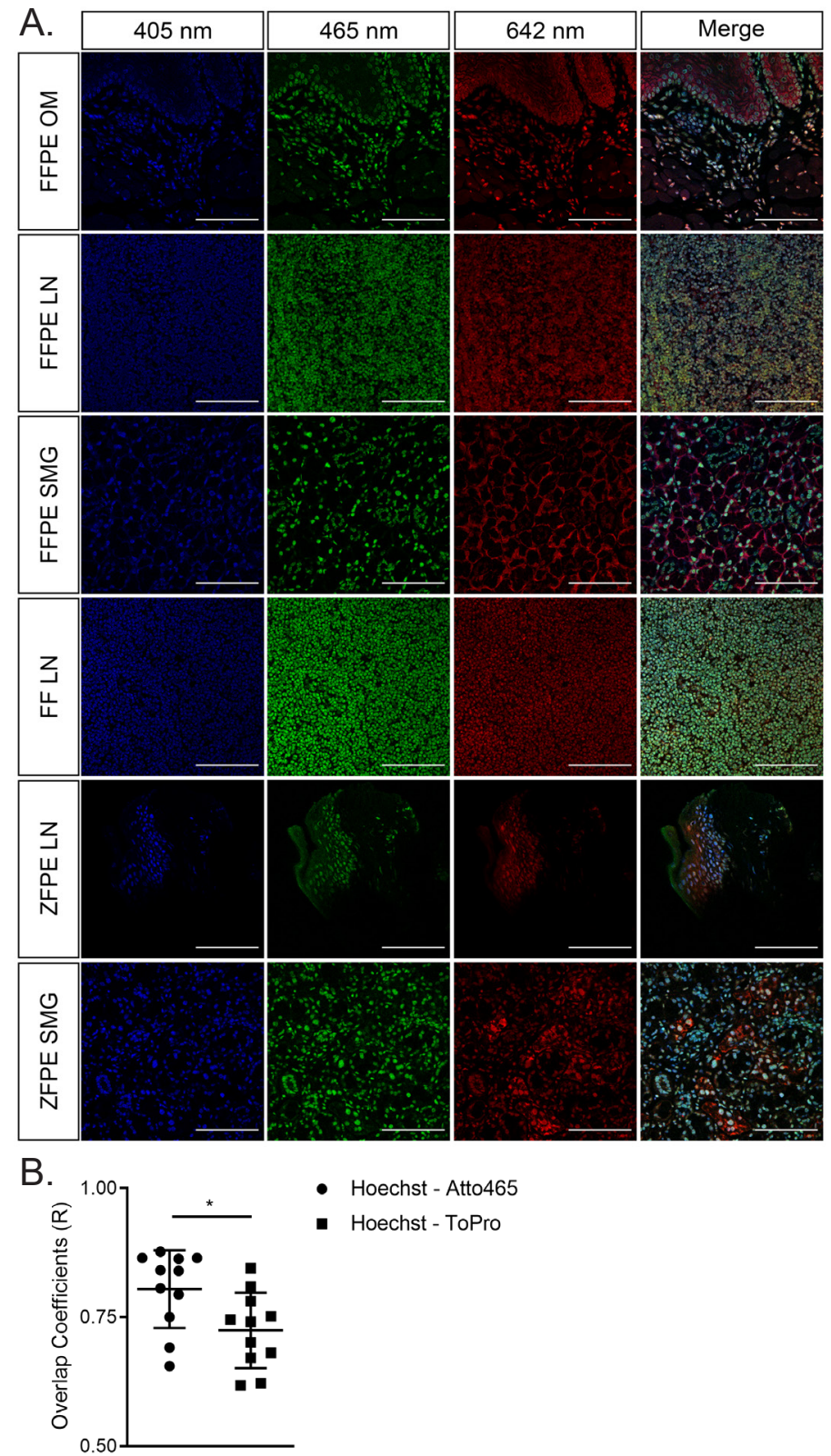

Figure 5: A) Mouse tissues following different fixation protocols to compare nuclear specificity of Atto 465-p to Hoechst 33342 and ToPro-3. OM = Oral mucosa; $\mathrm{LN}=$ lymph nodes; $\mathrm{SMG}=$ submandibular gland; ZFPE = zinc-fixed paraffin-embedded; FF - flash-frozen; Scale bar $=100 \mu \mathrm{m}$. B) Quantification of the overlap coefficient demonstrated higher similarity between Hoechst 33342 and Atto 465-p than Hoechst 33342 and ToPro-3. Values represent mean $+/$-SEM. * $=p<0.001$, paired t-test.

we conducted chromosomal staining using Hoechst 33342 as the positive control. Here, Atto 465-p demonstrated similar staining patterns with Hoechst 33342, suggesting that Atto 465-p stains chromatin (Fig. 4).

Atto 465-p stains nuclei in various cell types and conditions, making available a novel fluorescent channel for mIF protocols

To further assess potential applications for Atto 465-p as a nuclear dye, several types of mouse (Figure 5A) and human (Figure S2) sections of tissues fixed with three disparate processing protocols were subsequently stained with Atto 465-p, Hoechst 33342 or ToPro-3,. Figures 5A and S2 

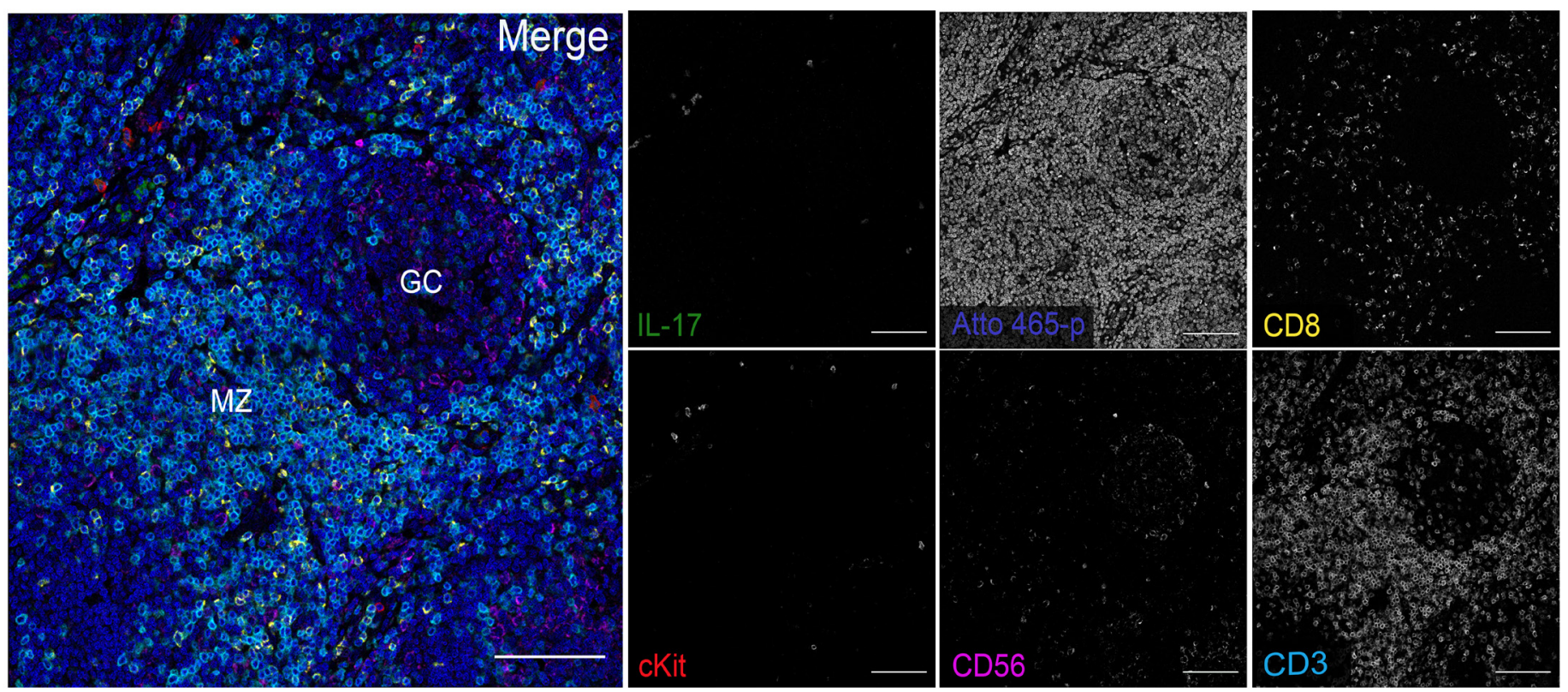

Figure 6: Atto 465-p as a nuclear dye. mIF microscopy of IL-17 (green), CD8 (yellow), cKit (red) CD56 (pink), and CD3 (cyan) in FFPE human tonsil. Atto $465-p$ was used as a nuclear probe and shows distinguishable staining from the $405 \mathrm{~nm}$ and $488 \mathrm{~nm}$ channels. $\mathrm{GC}=$ germinal center; $\mathrm{MZ}=$ marginal zone. Scale bar $=100 \mu \mathrm{m}$.

show a nearly identical staining pattern of Atto 465-p, Hoechst Discussion

33342 and ToPro-3 in all tissues, independent of tissue fix- In the present work, we demonstrate that Atto 465-p is a ation protocol. Quantitative colocalization analysis (Figure specific and stable fluorescent dye for nuclear staining with 5B and Supplementary Table 2) demonstrated higher degree excellent performance. It can be used as an alternative to of colocalization between Hoechst 33342 and Atto 465-p (R DAPI in mIF assays in mouse and human cells and tissues. $=0.8043+/-0.022)$ than Hoechst 33342 and ToPro-3 $(R=$ These data show comparable nuclear staining between Atto $0.7242+/-0.022)$. 465-p and other common nuclear dyes Hoechst 33342 and ToPro-3.

To test if Atto 465-p is suitable for mIF, we developed a 6-plex TSA panel using Atto 465-p as a nuclear probe. Human tonsil was stained for known surface markers, CD3 (T cells), CD8 ( $T$ cell subset), cKit (mast/innate lymphoid cells) and CD56 (NK cells) as well as an intracellular cytokine, IL-17, and visualized by confocal microscopy (Figure 6). Atto 465-p staining (shown in blue) highlights the border of each cellular nucleus, revealing the marginal zone (MZ) and germinal center (GC) of a follicle. As expected, CD3, CD8 and cKit showed membranous staining, residing primarily extrafollicularly, whereas CD56 expression was membranous but present both in the extra and intrafollicular space. Moreover, IL-17 cytoplasmic staining was localized primarily outside the germinal center. As a proof of concept, each channel was evaluated individually showing that Atto 465-p can be fluorescently separated from the 405nm (IL-17 green) and 488nm (CD8 yellow) channels. Together, these data suggest that Atto 465-p can be added as a nuclear stain to a $\mathrm{mIF}$ protocol, thus facilitating the use of the $405 \mathrm{~nm}$ wavelength for another target.

Atto 465-p is a green fluorescent dye that absorbs light between 439 and $465 \mathrm{~nm}$ (Figure 1). It is easily excited with an argon laser in instruments emitting 488-nm light, which is a more common imaging component than is the UV laser. The spectral properties of Atto 465-p favor its color separation from Alexa Fluor 405 and Alexa Fluor 488 (Figure 6), which provides a stable and specific alternative to leave the $405 \mathrm{~nm}$ channel open for additional targets when spectral imaging or a white light laser are available.

Importantly, UV excitation can be more toxic to cells than visible light (19), which can hinder further applicability in live cell microscopy studies. Our results demonstrate that extended exposure to blue light $(486 \mathrm{~nm})$ will prompt a photoconversion reaction whose product absorbs $405 \mathrm{~nm}$ light and emits blue fluorescence. Photoconversion has been shown in other common nuclear dyes like DAPI and Hoechst 33342 (20), although such event happened after high levels of energy were concentrated on the dye. A single image scan at normal settings is about $0.23 \mathrm{~nJ} / \mu \mathrm{m} 2$, a much lower amount than what was used in these photobleaching experiments (Figure 2 ). While this photoconversion is a cautionary characteristic for IHC multiplexing, the same characteristic has been leveraged in experiments as a simple method to mark specific cells (21). It should be noted that the amount of energy used to bleach and photoconvert Atto 465p is much higher 


\section{ARTICLE PREPRINT}

than typical confocal microscope settings for mIF. However, further investigation into the potential of Atto 465-p for live imaging is warranted. Leveraging of Atto 465-p as a photoinducible cell marker would be advantageous to the field, given that photoinducible markers, such as KikGR (22), PS-CFP (23), PA-GFP (24), PA-mRFP1 (25) and others, require ultraviolet light.

Taken together, Atto 465-p demonstrates both specificity and photostability and fulfils the requirements to be used as a nuclear dye for immunocytochemistry and immunohistochemistry applications. The use of Atto 465-p for nuclear staining increases the multiplex capacity in mIF assays by occupying an underutilized region in the fluorescent spectrum.

\section{Acknowledgements}

This work was funded by the intramural program of the National Institute of Dental and Craniofacial Research, National Institutes of Health. Human karyotype slides were kindly prepared and provided by National Human Genome Research Institute Cytogenetic and Microscopy Core. This research was supported by the NIDCR Imaging Core: ZIC DE000750. The authors thank Dr. Andrew Doyle for his technical expertise and review of the manuscript.

\section{References}

1. Sood A, Miller AM, Brogi E, Sui Y, Armenia J, McDonough E, et al. Multiplexed immunofluorescence delineates proteomic cancer cell states associated with metabolism. JCI Insight. 2016;1(6). doi: 10.1172/jci.insight.87030. PubMed PMID: 27182557; PubMed Central PMCID: PMCPMC4863708.

2. Gorris MAJ, Halilovic A, Rabold K, van Duffelen A, Wickramasinghe IN, Verweij D, et al. Eight-Color Multiplex Immunohistochemistry for Simultaneous Detection of Multiple Immune Checkpoint Molecules within the Tumor Microenvironment. J Immunol. 2018;200(1):347-54. Epub 2017/11/15. doi: 10.4049/jimmunol.1701262. PubMed PMID: 29141863.

3. Hofman P, Badoual C, Henderson F, Berland L, Hamila M, Long-Mira E, et al. Multiplexed Immunohistochemistry for Molecular and Immune Profiling in Lung Cancer-Just About Ready for Prime-Time? Cancers (Basel). 2019;11(3). Epub 2019/02/27. doi: 10.3390/cancers11030283. PubMed PMID: 30818873; PubMed Central PMCID: PMCPMC6468415.

4. Goto S, Morigaki R, Okita S, Nagahiro S, Kaji R. Development of a highly sensitive immunohistochemical method to detect neurochemical molecules in formalin-fixed and paraffin-embedded tissues from autopsied human brains. Front Neuroanat. 2015;9:22. Epub 2015/03/03. doi: 10.3389/fnana.2015.00022. PubMed PMID: 25784860; PubMed Central PMCID: PMCPMC4347496.

5. Bogoslovsky T, Bernstock JD, Bull G, Gouty S, Cox BM, Hallenbeck JM, et al. Development of a systems-based in situ multiplex biomarker screening approach for the assessment of immunopathology and neural tissue plasticity in male rats after traumatic brain injury. J Neurosci Res. 2018;96(4):487-500. Epub 2017/05/02. doi: 10.1002/ jnr.24054. PubMed PMID: 28463430; PubMed Central PMCID: PMCPMC5668208.

6. Dixon AR, Bathany C, Tsuei M, White J, Barald KF, Takayama S. Recent developments in multiplexing techniques for immunohistochemistry. Expert Rev Mol Diagn. 2015;15(9):1171-86. Epub 2015/08/06. doi: 10.1586/14737159.2015.1069182. PubMed PMID: 26289603; PubMed Central PMCID: PMCPMC4810438.

7. Saka SK, Wang Y, Kishi JY, Zhu A, Zeng Y, Xie $W$, et al. Immuno-SABER enables highly multiplexed and amplified protein imaging in tissues. Nat Biotechnol. 2019;37(9):1080-90. Epub 2019/08/19. doi: 10.1038/ s41587-019-0207-y. PubMed PMID: 31427819; PubMed Central PMCID: PMCPMC6728175.

8. Goltsev Y, Samusik N, Kennedy-Darling J, Bhate S, Hale M, Vazquez G, et al. Deep Profiling of Mouse Splenic Architecture with CODEX Multiplexed Imaging. Cell. 2018;174(4):968-81.e15. Epub 2018/08/02. doi: 10.1016/j. cell.2018.07.010. PubMed PMID: 30078711; PubMed Central PMCID: PMCPMC6086938.

9. Wang G, Achim CL, Hamilton RL, Wiley CA, Soontornniyomkij V. Tyramide signal amplification method in multiple-label immunofluorescence confocal microscopy. Methods. 1999;18(4):459-64. doi: 10.1006/meth.1999.0813. PubMed PMID: 10491275.

10. Toth ZE, Mezey E. Simultaneous visualization of multiple antigens with tyramide signal amplification using antibodies from the same species. J Histochem Cytochem. 2007;55(6):545-54. Epub 2007/01/24. doi: 10.1369/ jhc.6A7134.2007. PubMed PMID: 17242468.

11. Macechko PT, Krueger L, Hirsch B, Erlandsen SL. Comparison of immunologic amplification vs enzymatic deposition of fluorochrome-conjugated tyramide as detection systems for FISH. J Histochem Cytochem. 1997;45(3):359-63. doi: 10.1177/002215549704500303. PubMed PMID: 9071317.

12. van Gijlswijk RP, Zijlmans HJ, Wiegant J, Bobrow MN, Erickson TJ, Adler KE, et al. Fluorochrome-labeled tyramides: use in immunocytochemistry and fluorescence in situ hybridization. J Histochem Cytochem. 1997;45(3):37582. doi: 10.1177/002215549704500305. PubMed PMID: 9071319.

13. Arden-Jacob J, Drexhage KH, Druzhinin SI, Ekimova M, Flender O, Lenzer T, et al. Ultrafast photoinduced dynamics of the 3,6-diaminoacridinium derivative ATTO 465 in solution. Phys Chem Chem Phys. 2013;15(6):184453. Epub 2012/12/19. doi: 10.1039/c2cp43493h. PubMed PMID: 23247684.

14. Doyle AD, Wang FW, Matsumoto K, Yamada KM. One-dimensional topography underlies three-dimensional fibrillar cell migration. J Cell Biol. 2009;184(4):481-90. Epub 2009/02/18. doi: 10.1083/jcb.200810041. PubMed PMID: 19221195; PubMed Central PMCID: PMCPMC2654121.

15. Feng Z, Jensen SM, Messenheimer DJ, Farhad 
$M$, Neuberger $M$, Bifulco $C B$, et al. Multispectral Imaging of $\mathrm{T}$ and $\mathrm{B}$ Cells in Murine Spleen and Tumor. J Immunol. 2016;196(9):3943-50. Epub 2016/03/20. doi: 10.4049/jimmunol.1502635. PubMed PMID: 26994219; PubMed Central PMCID: PMCPMC4834492.

16. Costes SV, Daelemans D, Cho EH, Dobbin Z, Pavlakis G, Lockett S. Automatic and quantitative measurement of protein-protein colocalization in live cells. Biophys J. 2004;86(6):3993-4003. Epub 2004/06/11. doi: 10.1529/biophysj.103.038422. PubMed PMID: 15189895; PubMed Central PMCID: PMCPMC1304300.

17. Hermanson GT. Bioconjugate techniques: Academic press; 2013.

18. Suzuki T, Fujikura K, Higashiyama T, Takata K. DNA staining for fluorescence and laser confocal microscopy. J Histochem Cytochem. 1997;45(1):49-53. doi: 10.1177/002215549704500107. PubMed PMID: 9010468.

19. GeJ, Wood DK, WeingeistDM, PrasongtanakijS, Navasumrit $\mathrm{P}$, Ruchirawat $\mathrm{M}$, et al. Standard fluorescent imaging of live cells is highly genotoxic. Cytometry A. 2013;83(6):552-60. Epub 2013/05/06. doi: 10.1002/cyto.a.22291. PubMed PMID: 23650257; PubMed Central PMCID: PMCPMC3677558.

20. Karg TJ, Golic KG. Photoconversion of DAPI and Hoechst dyes to green and red-emitting forms after exposure to UV excitation. Chromosoma. 2018;127(2):235-45. Epub 2017/12/12. doi: 10.1007/s00412-017-0654-5. PubMed PMID: 29234867 ; PubMed Central PMCID: PMCPMC5945337.

21. Ando R, Hama $\mathrm{H}$, Yamamoto-Hino M, Mizuno $\mathrm{H}$, Miyawaki A. An optical marker based on the UV-induced greento-red photoconversion of a fluorescent protein. Proc Natl Acad Sci U S A. 2002;99(20):12651-6. Epub 2002/09/23. doi: 10.1073/pnas.202320599. PubMed PMID: 12271129; PubMed Central PMCID: PMCPMC130515.

22. Tsutsui H, Karasawa S, Shimizu H, Nukina N, Miyawaki A. Semi-rational engineering of a coral fluorescent protein into an efficient highlighter. EMBO Rep. 2005;6(3):233-8. doi: 10.1038/sj.embor.7400361. PubMed PMID: 15731765; PubMed Central PMCID: PMCPMC1299271.

23. Chudakov DM, Verkhusha VV, Staroverov DB, Souslova EA, Lukyanov S, Lukyanov KA. Photoswitchable cyan fluorescent protein for protein tracking. Nat Biotechnol. 2004;22(11):1435-9. Epub 2004/10/24. doi: 10.1038/nbt1025. PubMed PMID: 15502815.

24. Patterson GH, Lippincott-Schwartz J. A photoactivatable GFP for selective photolabeling of proteins and cells. Science. 2002;297(5588):1873-7. doi: 10.1126/science.1074952. PubMed PMID: 12228718.

25. Verkhusha VV, Sorkin A. Conversion of the monomeric red fluorescent protein into a photoactivatable probe. Chem Biol. 2005;12(3):279-85. doi: 10.1016/j.chembiol.2005.01.005. PubMed PMID: 15797211. 
bioRxiv preprint doi: https://doi.org/10.1101/2021.05.14.444234; this version posted May 15, 2021. The copyright holder for this preprint (which was not certified by peer review) is the author/funder. This article is a US Government work. It is not subject to copyright under 17 USC 105 and is also made available for use under a CCO license.

Atto465 derivative is a unique nuclear stain

\section{Supplementary Data}

Supplementary Table 1: List of antibodies used for mIF

\begin{tabular}{|c|c|c|c|c|}
\hline Type & $\begin{array}{l}\text { Antibody name } \\
\text { and catalogue } \\
\text { number }\end{array}$ & Dilution & Source & Cell Target \\
\hline \multirow{5}{*}{$\begin{array}{l}\text { Primary } \\
\text { antibody }\end{array}$} & rat anti-CD3 & $1: 2000$ & BioRad, Hercules, CA & T cells \\
\hline & mouse anti-CD8 & 1:1000 & BioRad & T cell subset \\
\hline & rabbit anti-CD56 & $1: 1000$ & Abcam, Cambridge, MA & Natural killer cells \\
\hline & rabbit anti-cKit & 1:1000 & Abcam & $\begin{array}{l}\text { Mast cell / Innate } \\
\text { Lymphoid cell }\end{array}$ \\
\hline & goat anti-IL-17 & $1: 1000$ & $\begin{array}{c}\text { R\&D Systems, Minneapolis, } \\
\text { MN }\end{array}$ & IL-17 producing cells \\
\hline \multirow{5}{*}{$\begin{array}{c}\text { HRP- } \\
\text { conjugated } \\
\text { Tyramide } \\
\text { antibodies }\end{array}$} & Alexa Fluor 405 & $1: 500$ & \multirow{4}{*}{$\begin{array}{c}\text { Thermo-Fisher Scientific, } \\
\text { Eugene, OR }\end{array}$} & - \\
\hline & Alexa Fluor 488 & $1: 200$ & & - \\
\hline & Alexa Fluor 555 & $1: 100$ & & - \\
\hline & Alexa Fluor 594 & $1: 100$ & & - \\
\hline & Atto 633 & $1: 500$ & $\begin{array}{c}\text { Sigma-Aldrich, St. Louis, } \\
\text { MO }\end{array}$ & - \\
\hline
\end{tabular}

Supplementary Table 2: Overlap Coefficient - Figure 5

\begin{tabular}{|l|l|l|}
\hline Tissue & $\begin{array}{l}\text { Hoechst 33342 to Atto 465-p } \\
\text { Overlap Coefficient (R) }\end{array}$ & $\begin{array}{l}\text { Hoechst 33342 to ToPro-3 } \\
\text { Overlap Coefficient (R) }\end{array}$ \\
\hline Human FFPE Tonsil & 0.865 & 0.781 \\
\hline Human FFPE MSG & 0.865 & 0.845 \\
\hline Human FFPE Oral Mucosa & 0.691 & 0.622 \\
\hline Human FFPE Skin & 0.750 & 0.701 \\
\hline Human FFPE Kidney & 0.655 & 0.618 \\
\hline Mouse FFPE Oral Mucosa & 0.794 & 0.752 \\
\hline Mouse FFPE LN & 0.863 & 0.809 \\
\hline Mouse FFPE SMG & 0.806 & 0.681 \\
\hline Mouse Frozen Lymph Node & 0.877 & 0.745 \\
\hline Mouse Zinc Lymph Node & 0.840 & 0.741 \\
\hline Mouse Zinc SMG & 0.841 & 0.671 \\
\hline
\end{tabular}


Atto465 derivative is a unique nuclear stain

A

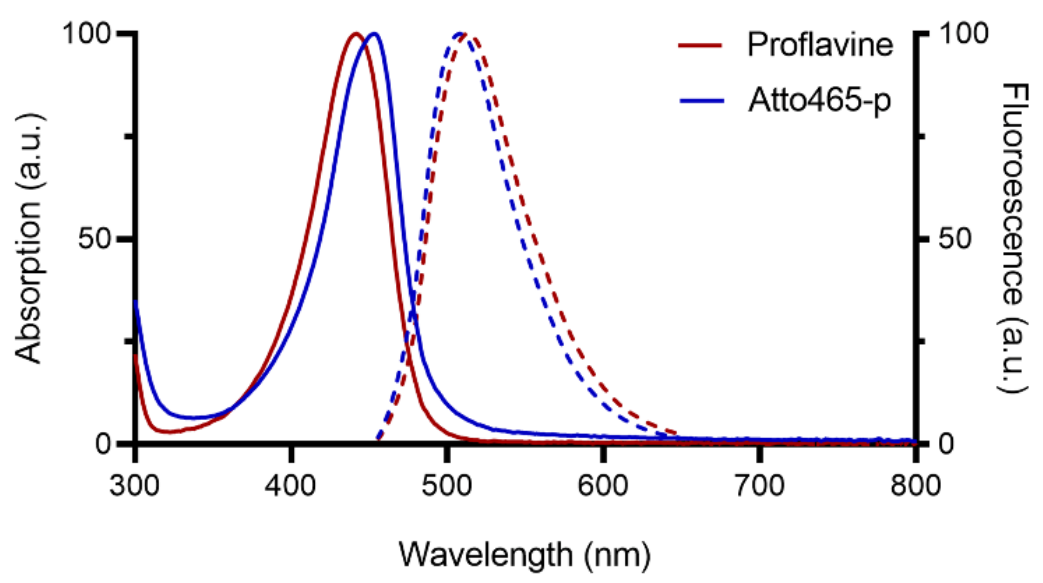

B
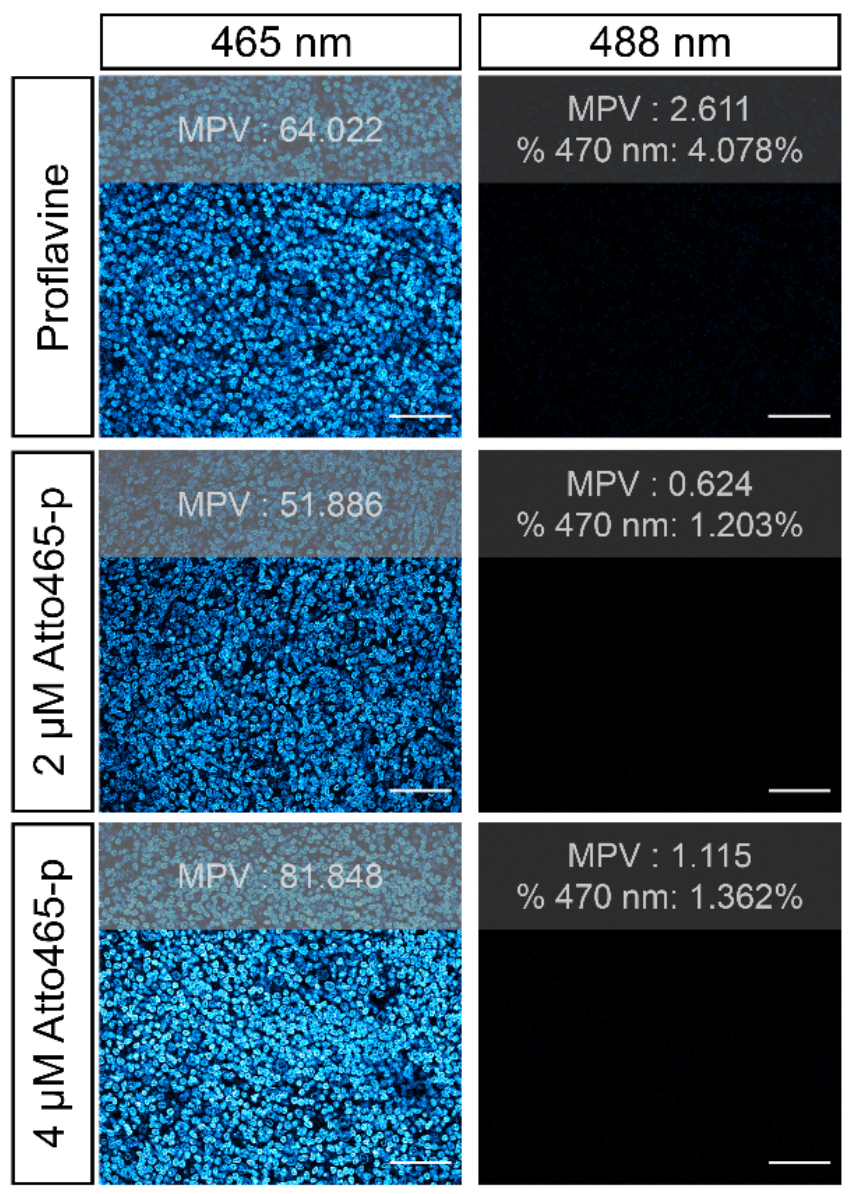

Supplemental Figure 1: A) Absorption (solid line) and fluorescence emission (dashed line) spectra of 8

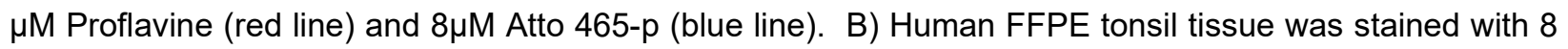
$\mu \mathrm{M}$ Proflavine, $2 \mu \mathrm{M}$ or $4 \mu \mathrm{M}$ of Atto 465-p fluorescence signal measured following excitation at $465 \mathrm{~nm}$ and $488 \mathrm{~nm}$ on a Leica SP8 using the same microscope settings. Images were converted to Cyan Hot lookup table (LUT) to better demonstrate staining intensities. Proflavine shows higher background and fluorescent bleed-through into $488 \mathrm{~nm}$ than Atto465-p. Scale bar $=25 \mu \mathrm{m}$ 
bioRxiv preprint doi: https://doi.org/10.1101/2021.05.14.444234; this version posted May 15, 2021. The copyright holder for this preprint (which was not certified by peer review) is the author/funder. This article is a US Government work. It is not subject to copyright under 17 USC 105 and is also made available for use under a CCO license.

Atto465 derivative is a unique nuclear stain

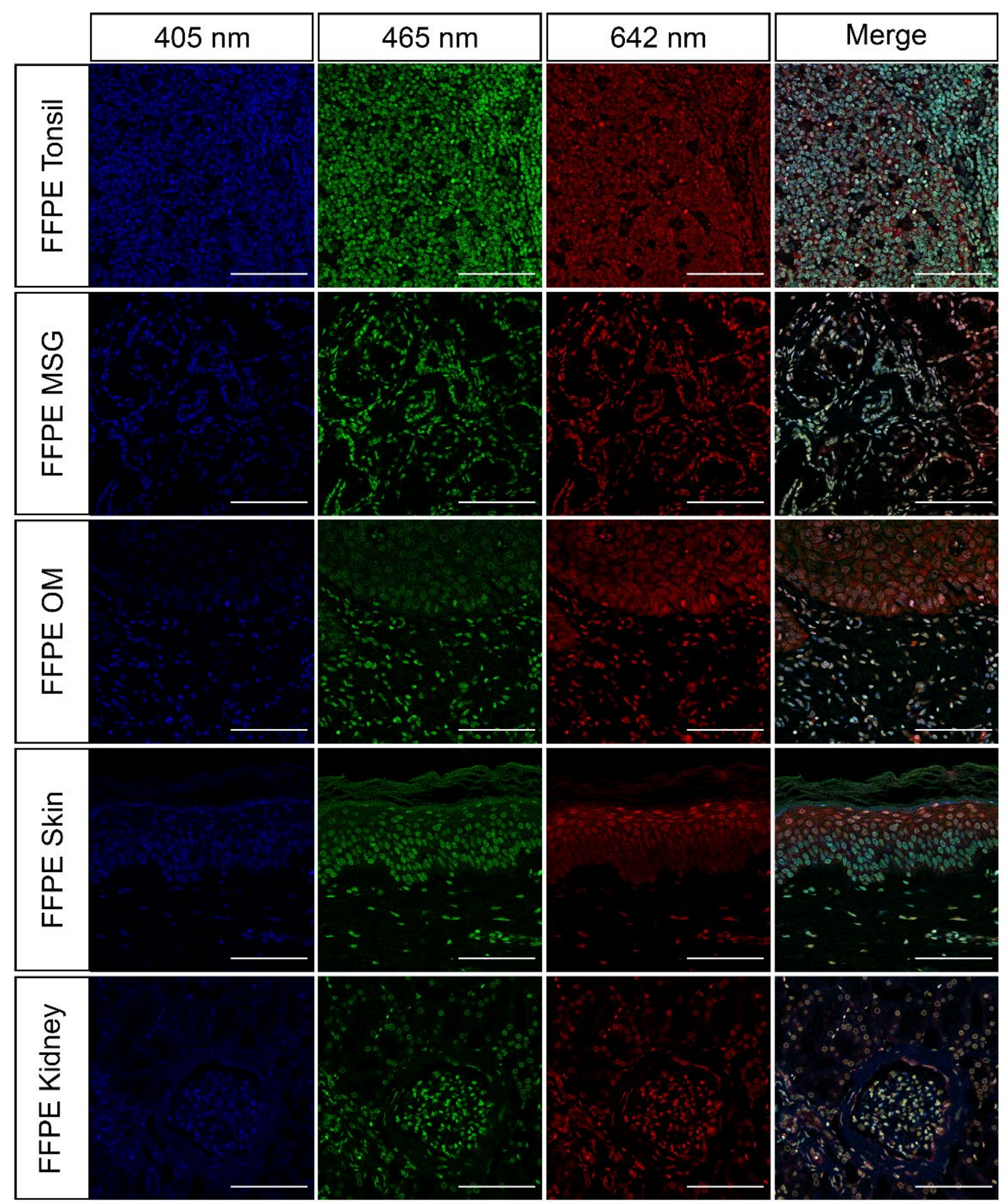

Supplemental Figure 2: Human FFPE tonsil, MSG, OM, skin, and kidney were stained with Hoechst $(16.2 \mu \mathrm{M})$ and ToPro $(1 \mu \mathrm{M})$ and imaged on a Nikon confocal microscopy to demonstrate similar nuclear specificity of Atto $465-p(4 \mu \mathrm{M})$. Scale bar $=100 \mu \mathrm{m}$. 